\title{
Nonlinear properties of optically dense medium excited by multifrequency laser radiation
}

\author{
Konstantin Barantsev*, Andrey Litvinov, and Evgeniy Popov \\ Peter the Great St. Petersburg Polytechnic University, Department of theoretical physics, \\ 29 Polytechnicheskaia str., Saint-Petersburg, 195251 Russia
}

\begin{abstract}
This work is devoted to nonlinear effects in the atomic medium excited by multifrequency laser radiation. We have considered collective light scattering in the optically dense medium in conditions of coherent population trapping. Propagation of intermode correlation coefficient was calculated and it was found that correlation can increase in the medium. Light shifts of the coherent population trapping resonances and ways of their suppression were analyzed. Propagation of radiation in the medium with closed excitation scheme was investigated.
\end{abstract}

Optical properties of an atomic medium can sufficiently depend on parameters of the light which is scattered in this medium. This fact is the appearance of nonlinear effects. So, under the conditions of electromagnetically induced transparency the medium with the $\Lambda$-scheme of energy levels becomes transparent for the probe pulse on one of the transitions when the control field interacts with the second transition [1]. Such transparence appears due to destructive quantum interference between the two channels of excitation in the $\Lambda$-scheme, that is known as coherent population trapping (CPT) $[2,3]$. It is possible to control this effect (and therefore the transparency of the medium) by variation frequency detuning or intensities of the field.

However, in optically dense medium there is changing in the parameters of radiation due to absorption and scattering of the last one, and the atoms of deeper layers interacts with modified radiation. Especially, the intensity, spectrum, initial phase and statistics can change in the space. In the case of the radiation with several carrier frequencies, which is used for excitation the CPT resonance, the cross-correlation between frequency components can be modified. Such phenomena, when light scattering on a single atom depends on scattering on the other atoms, are called collective effects [4,5].

This work is devoted to theoretical investigation of the collective light scattering by optically dense atomic medium under conditions of the CPT resonance. Topicality is due to using of CPT in such areas as quantum frequency standards [3], magnetometry [6,7], quantum memory and deceleration of light $[8,9]$, etc. Propagation of cross-correlations between the modes of the radiation is investigated in the optically dense gas of the alkali atoms. It was found that level of correlations can increase when the radiation passes through the medium.

Corresponding author: kostmann@yandex.ru 
We analyzed the light shifts of the CPT resonance with the view to reducing their sensitivity to intensity fluctuations. The intensity ratio of the frequency components, which provides the lowest sensitivity of the light shift to changing in the total intensity, was found in the optically dense medium.

Particular attention is drawn to the mediums with a closed excitation scheme excited by the laser radiation. Under these conditions the optical properties of the medium, especially CPT state, depend on initial phases of the radiation frequency components. It is possible both to destroy or restore CPT state by variation of the initial phases $[10,11]$. The paper [12] demonstrates the possibility of controlling absorption and refraction of the medium by means of changing in the initial phase of radiation.

In our work we calculate propagation of the radiation in the optically dense medium in the case of closed excitation scheme. We demonstrate that it is possible to control the space distribution of the absorption and refraction of the medium by changing in the initial phases of the frequency components of the radiation.

The work was supported by the Russian Science Foundation (project 17-12-01085).

\section{References}

[1] M. Fleischhauer, A. Imamoglu, J.P. Marangos, Rev. Mod. Phys. 77, 633 (2005)

[2] E. Arimondo, Progress in optics 35, 259 (1996)

[3] J. Vanier, Appl. Phys. 81, 421, (2005)

[4] C.C. Kwong, T. Yang, D. Delande et al., Phys. Rev. Lett. 115, 223601 (2015)

[5] V.M. Datsyuk, I.M. Sokolov, D.V. Kupriyanov et al., Phys. Rev. A 77, 033823 (2008)

[6] M. Stahler, R. Wynands, S. Knappe et al., Opt. Lett. 27, 1472 (2002)

[7] A. Akulshin, A. Celikov, V. Velichansky, Opt.Commun. 84, 139 (1991)

[8] M.D. Lukin, Rev. Mod. Phys. 75, 457 (2003)

[9] A. Imamoglu, J.P. Marangos, Rev. Mod. Phys. 77, 633 (2005)

[10] D.V. Kosachiov, B.G. Matisov, Yu.V. Rozhdestvensky, J. Phys. B: At. Mol. Opt. Phys. 25, 2473 (1992)

[11] S.J. Buckle, S.M. Barnett, P.L. Knight et al., Optica Acta 33, 1129 (1986)

[12] H. Li, V.A. Sautenkov, Yu.V. Rostovtsev et al., Phys. Rev. A 80, 023820 (2009) 